\title{
The Effectiveness of Infection Control Practices among Health Care Workers Responding to the COVID-19 Pandemic in Nigeria
}

\author{
Fehintola A. Ige1 ${ }^{*}$, Aigbe G. Ohihoin'2, Bosede 0. Amuda ${ }^{3}$, Olufemi S. Amoo', Chika K. Onwuamah', \\ Azuka P. Okwuraiwe1, Joseph 0. Shaibu' ${ }^{1}$, Ebenezer 0. Odewale ${ }^{1}$, Ayorinde B. James ${ }^{4}$, \\ Adeleke Kayode', Adefunke Adeshina ${ }^{6}$, Rosemary A. Audu' ${ }^{1}$ \\ ${ }^{1}$ Centre for Human Virology and Genomics, Department of Microbiology, Nigerian Institute of Medical Research, Lagos, Nigeria \\ ${ }^{2}$ Clinical Science Department, Nigerian Institute of Medical Research, Lagos, Nigeria \\ ${ }^{3}$ Centre for Tuberculosis Research, Department of Microbiology, Nigerian Institute of Medical Research, Lagos, Nigeria \\ ${ }^{4}$ Biochemistry Department, Nigerian Institute of Medical Research, Lagos, Nigeria \\ ${ }^{5}$ Department of Community Health \& Primary Health Care, Lagos State University Teaching Hospital, Lagos, Nigeria \\ ${ }^{6}$ Lagos State Ministry of Health, Ikeja, Lagos, Nigeria \\ Email: ^fehin_ti@yahoo.com
}

How to cite this paper: Ige, F.A., Ohihoin, A.G., Amuda, B.O., Amoo, O.S., Onwuamah, C.K., Okwuraiwe, A.P., Shaibu, J.O., Odewale, E.O., James, A.B., Kayode, A., Adeshina, A. and Audu, R.A. (2021) The Effectiveness of Infection Control Practices among Health Care Workers Responding to the COVID-19 Pandemic in Nigeria. Advances in Infectious Diseases, 11, 232-239. https://doi.org/10.4236/aid.2021.112021

Received: March 19, 2021

Accepted: June 26, 2021

Published: June 29, 2021

Copyright $\odot 2021$ by author(s) and Scientific Research Publishing Inc. This work is licensed under the Creative Commons Attribution-NonCommercial International License (CC BY-NC 4.0). http://creativecommons.org/licenses/by-nc/4.0/ cC) (7) (9) Open Access

\begin{abstract}
Background: The world is currently experiencing a healthcare crisis caused by Severe Acute Respiratory Syndrome Coronavirus 2 (SARS-CoV-2). Healthcare workers (HCWs) are at the forefront of controlling the spread of the disease; this response has resulted in a huge number of infections amongst HCWs and unfortunately some casualties. Infection prevention and control practices (IPC) are practical, proven methods that prevent avoidable harm to patients and protect health workers from contagious infection. This study was done to evaluate the effectiveness of IPC practices utilized in COVID-19 testing drive through facility in Lagos Nigeria. Method: The facility recruited 42 HCWs who were trained on IPC and use of Personal Protective Equipment (PPEs). Training on donning and doffing of PPEs, preparation of decontaminants and sample handling were done for relevant personnel. A daily log for monitoring COVID-19 symptoms was deployed to all HCWs; to help early detection of COVID-19 infection and prevent transmission amongst staff. Results: Of 42 personnel, $92.8 \%$ had a tertiary level of education while $71.4 \%$ had at least 5 years work experience. A total of 5 (11.9\%) out of 42 HCWs were positive by week six of the study. All infected persons worked at the drive through centre. Most common symptom filled in the daily log tool was headache and fatigue. Conclusion: Despite all the measures introduced, $11.9 \%$ of HCWs became positive within 6 weeks, showing the highly infectious nature of the virus and the need to research into more effective meas-
\end{abstract}


ures in preventing the transmission of highly infectious pathogens to HCWs during outbreaks.

\section{Keywords}

Healthcare Workers, Infection Prevention and Control, COVID-19 Pandemic

\section{Introduction}

The Severe Acute Respiratory Syndrome Coronavirus 2 (SARS-CoV-2) is a novel coronavirus and the causative organism for the corona virus disease 2019 (COVID-19) which is responsible for the current global pandemic [1]. This highly contagious disease outbroke in Wuhan, China in December 2019, and the first COVID-19 confirmed case in Nigeria was reported on $27^{\text {th }}$ February 2020 [2]. The first COVID-19 confirmed case in Nigeria was reported on $27^{\text {th }}$ February 2020 [2]. COVID-19 is spread by human-to-human transmission through contact and droplets exposure. Symptoms include fever, fatigue, sore throat, cough, abdominal pain and dyspnea in mild cases and bilateral viral pneumonia, and acute respiratory distress syndrome (ARDS) in more severe cases [1].

Establishing measures to control the transmission of SARS-CoV-2 is a key public healthcare intervention goal. Healthcare workers (HCWs) are at the forefront of controlling the spread of COVID-19; this response and persistent exposure to infected patients and contaminated surfaces can increase the risk of acquiring and transmitting the infection [3]. Epidemiological data in Italy shows that as of $3^{\text {rd }}$ April 2020, around 10,000 healthcare workers had been infected and 74 had died [4]. In China, 3387 healthcare workers were infected [5]. This data highlights the importance of controlling the spread of the infection to HCWs.

In order to join global efforts to contain and defeat the pandemic, Nigeria established its first modified drive through testing facility (MDTF) in Nigeria Institute of Medical Research (NIMR) Lagos on the $30^{\text {th }}$ of March 2020 [6]. A drive through testing strategy is an innovative way of increasing testing while reducing infection transmission; it has the advantage of reduced contact and exposure time with clients [7]. Standard IPC measures and controls were introduced to ensure that HCWs were protected from infection in this scheme. The objective of the study is to determine the effectiveness of IPC measures deployed in the implementation of the drive-through testing strategy at NIMR.

\section{Methods}

\subsection{Study Site}

This study was coordinated by the Centre for Human Virology and Genomics, Nigeria Institute of Medical Research, Lagos. The centre is an ISO 15189:2012 accredited laboratory and listed as a World Health Organization (WHO) pre- 
qualification evaluating laboratory.

\subsection{Study Design and Participants}

The study is a mixed method study involving qualitative and quantitative components; the use of in-depth interview was employed for the qualitative aspects and summary statistics for quantitative analysis.

The MDTF recruited HCWs (referred to as the NIMR COVID-19 response team) from various health multicentres in the Lagos province. This team had 42 personnel comprised of clinicians, researchers, scientists, technicians, environmental health officers and other administrative staff (Table 1) who were assigned into various sub groups such as the surveillance team, sample collection, laboratory testing, viral transport medium \& decontamination team, data entry\& result validation team, sanitation and administrative teams. The MDTF had a modification of a walk-in pathway introduced to accommodate walk-in clients who do not own cars.

Prior to start up, risk assessment was conducted using a standardized template from WHO and gaps identified were closed [8]. All HCWs were trained on Infection Prevention and Control practices by the IPC and biosecurity team in the institute using the institutes biosafety manual and standard operating procedures. A daily log for monitoring COVID-19 symptoms was developed. The response team was trained on how to complete the daily log which was by ticking symptoms (cough, fever, abdominal pain, diarrhoea, rhinorhea) they could be experiencing. The purpose of this daily log was to aid early detection of COVID-19 infection should that occur. HCWs were encouraged to test when they had symptoms suggestive of COVID-19 or before visiting their families as they were lodged onsite.

Table 1. Distribution of health care workers recruited.

\begin{tabular}{|c|c|c|c|}
\hline Team & Number & Educational Qualification & Years of experience \\
\hline \multirow{3}{*}{ Surveillance } & 4 & Tertiary & $10-15$ years \\
\hline & & & \\
\hline & 2 & Tertiary & $0-5$ years \\
\hline \multirow{2}{*}{ Sample Collection } & 5 & Tertiary & $10-15$ years \\
\hline & 2 & Tertiary & $0-5$ years \\
\hline \multirow{2}{*}{ Laboratory Testing } & 7 & Tertiary & $10-15$ years \\
\hline & 2 & Tertiary & $0-5$ years \\
\hline Viral transport medium & 5 & Tertiary & 5 - 10 years \\
\hline \& decontaminant production & 5 & Tertiary & $0-5$ years \\
\hline Data entry \& Result validation & 3 & Tertiary & $10-15$ years \\
\hline Sanitation team & 3 & Secondary & $0-5$ years \\
\hline Administrative team & 4 & Tertiary & $15-20$ years \\
\hline
\end{tabular}


The activity workflow (Figure 1) for the testing facility went thus: at the MDTF, surveillance data and sample (oral and nasopharyngeal swab) were collected, the next step was to transport biological samples to a biosafety level 3 (BSL-3) laboratory for sample inactivation. After which samples were moved for qPCR testing in a biosafety level 2 (BSL-2) laboratory and finally, results were released via email after data entry and validation. The process for inactivation was by aliquoting samples into a lysis buffer and leaving to stand at room temperature for 10 minutes. Thereafter, the aliquot was moved to a BSL II (BSL2) laboratory for routine laboratory testing. Sample and data collection at the MDTF was from 10 am to 4:00 pm for six days a week and an average of 75 clients was serviced per day. The period reported in this study was from $30^{\text {th }}$ March to May $10^{\text {th }}$.

\section{Results}

There were $42 \mathrm{HCWs}$ aged between 21 years and 57 years, with a mean age of 32 years, comprising of 29 males and 13 females. Out of the 42 HCWs, 39 (92.8\%) attained tertiary level of education while three persons (7.1\%) attained secondary level of education. Fourteen of them (33.3\%) had work experience of less than 5 years, while 28 (66.6\%) had work experience of greater than 5 years. Of the 42 HCWs recruited in the study, 37 completed the study. Five HCWs who got infected were removed to isolation wards.

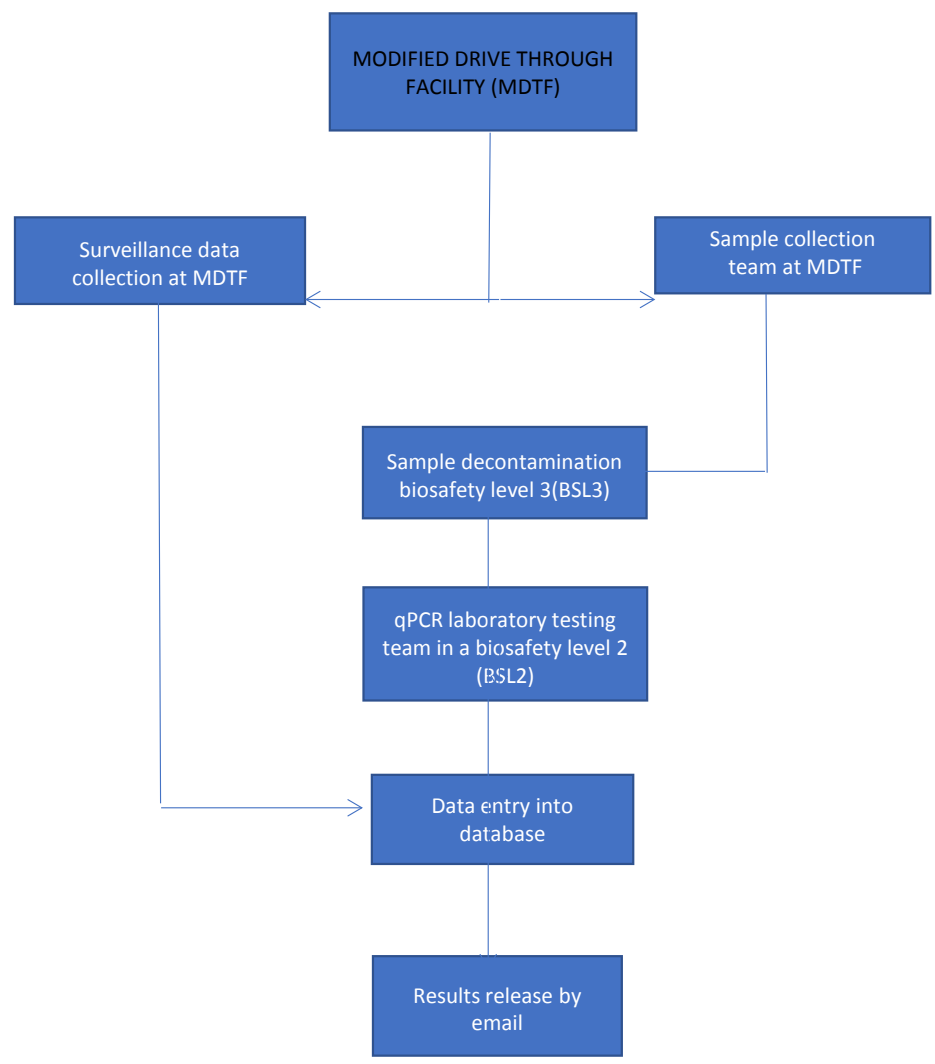

Figure 1. Activity workflow for modified drive through testing facility. 


\section{COVID-19 Testing Per Week}

Week 1: No member of the COVID-19 response team was tested in the first week as we had just commenced testing at the centre no staff had suggestive symptoms which required testing.

Week 2: Two onsite staff from the sample collection team working at the MDTF were tested; they indicated interest in visiting their families. Their result was negative. No other staff filled any suggestive symptom in the COVID daily $\log$ which required testing.

Week 3: The entire NIMR COVID response team (42) was tested; 3 personnel were positive. These personnels had not reported any symptoms in their daily log. All personnel infected were stationed at the MDTF (from the sample collection team and data surveillance team).

Week 4: No staff reported suggestive symptom, which required testing in the week.

Week 5: A team member from the sample collection unit reported fever, cough, rhinorrhea, muscular aches and fatigue to the biosafety officer during the week and was tested. The COVID-19 test result came out positive.

Week 6: A HCW with sample collection team tested positive after reporting headache, fatigue and muscular ache. All other team members in the sample collection team were offered testing and were negative for COVID- 19 .

A total of 5 (11.9\%) out of 42 HCWs were positive by week six. All infected persons worked at the MDTF; two of which were newly recruited volunteers.

\section{Discussion}

The study was conducted during a lockdown phase in the early stages of the COVID-19 outbreak when only 81 cases of COVID-19 had been reported as at March $30^{\text {th }}$, in Lagos Nigeria [2]. Some HCWS were lodged onsite to ensure uninterrupted testing services during the lockdown period and to prevent community infection. The above factors made it most likely that the affected healthcare workers were infected within the healthcare setting rather than from their household contacts. A total of five (11.9\%) out of the $42 \mathrm{HCWs}$ had tested positive at the sixth week, three were in the sample collection team and two were in the surveillance data team. All five positive personnel were noticed to work primarily at the MDTF. They were immediately isolated and evacuated within 5 hours of a positive test result to a COVID-19 isolation center for treatment and management Most of the HCWs that participated in the study were well trained, had a tertiary level of education and had more than five years working experience. Of the all the HCWs handling samples in the study (Figure 1) there was no infection recorded in persons that had no physical interaction with the MDTF. The laboratory testing team (Table 1) which handled the samples received from the MDTF after inactivation at the BSL3 laboratory, had 7 out of its 9 HCWs with greater than 10 years' work experience and were familiar with handling infectious agents. This was in line with other studies which had noted that a higher level of training, knowledge, and work experience increased conformity with 
IPC standard precautions and provided better outcome in avoiding infection [3] [6] [9].

The lack of infection reported in other teams working outside of the MDTF in our study may be due to the added precaution of inactivating all biological samples before testing in a Biosafety level III (BSL 3) laboratory (Figure 1) within the Institute. This added step may be responsible for interrupting infection and keeping other team members of the NIMR COVID-19 response team infection free.

Provision of a daily log of COVID-19 symptom record was a useful measure as this made HCWs consciously assess their health daily so as to pick subtle nuances in their wellbeing which helped in early detection of infection. This provided HCWs with an opportunity to discuss changes in their health quickly with the IPC officers who were in charge of tracking the completed logs.

Various interventions were launched to ensure the MDTF was infection free after confirmation of the first three cases (Week 3), the first of which was to shut down operations for 24 hours to allow for effective and thorough decontamination of the entire environment and retraining of all HCWs on IPC. The second intervention was a safety audit of the MDTF and the following were the gaps observed; inappropriate donning and interaction with PPEs, too many cars within the collection premises, inadequate personnel staffing and the long waiting time for walk-in clients making the activity slow and tedious, inadequate distance between points of sample collection and cars driving through, break time was not being observed as mandated due to the pressure of work.

Some other studies have noted that work pressure, intensity, and lack of rest increased the probability of infection for HCWs. In addition, inadequate training for IPC, particularly for respiratory-borne infectious diseases and high level of exposure and contact with infected persons also facilitated transmission of infection [1] [3] [6]. All of these were noticed in our study particularly in the MDTF where the intensity, infection exposure and pressure of work were high.

It is noteworthy that the HCWs wearing the full PPEs for sample collection reported that they were hot and uncomfortable adding a lot of stress to the normal issues of being fully gowned in PPE. This was because of the very hot and humid condition of the Nigerian climate, with an average temperature of $31^{\circ} \mathrm{C}$ $38^{\circ} \mathrm{C}$ in April [10]. It was observed that the humidity, hot weather and long hours of standing per shift during sample collection procedures led to sweating, fatigue and personnel fidgeting, interacting, and adjusting their PPEs more frequently than normal.

To correct the gaps identified a retraining on IPC was done for all HCWs during the $4^{\text {th }}$ week of the study. The training had an emphasis on respiratory-borne infectious disease (Infection Prevention and Control (IPC) for Novel Coronavirus (COVID-19), using the WHO training module for IPC for COVID-19 [11]. All participants had to provide a certificate of completion before they were allowed to resume. The sample collection team was also retrained on "How to put on and remove personal protective equipment"-COVID-19 using the WHO 
online training module [11]. To address the issues of break period, long hours of shift, infection exposure and PPE discomfort; the numbers of cars accessing the drive through were reduced to 4 per time to reduce the rate of exposure of HCWs to infected persons. Thirty minutes break period where no car or client was allowed to drive in and wait was re-emphasized, this was to allow for a rest period and a change of shift. Walk-in clients were allotted dedicated personnel to improve their waiting time.

HCWs at the drive through were advised to work in two shifts per day to allow for in between rest period and shorter time in full PPEs. Other teams were reassigned to allocate more members to the sample collection team due to shortfall in staff from the COVID-19 infection and to make room for 2 shifts. A weekly safety visit to the collection center to assess their compliance to best practices and quick intervention was also initiated.

A limitation was observed in the daily log for COVID-19 symptoms; at the time we started, the full spectrum of symptoms for the disease in our region of the world had not been fully described. The limitation is that there was no provision for "other" symptoms as a lot of HCWs had issues not described in the form. This shortfall was allayed by the discussion the IPC officers had with respondents in the course of monitoring the forms. Headache and fatigue were the most common complaints and this was noted for future tracking especially as two HCWS who tested positive had these symptoms.

The percentage of COVID-19 infection seen in this study (11.9\%) was higher than reported by other studies done in Iran where 5.6\% infection was reported and China $1.1 \%$ among HCWs, these two studies however sampled a larger population size 4854 and 9684 respectively [12] [13].

\section{Conclusion}

Despite all the preventive measures introduced, $11.9 \%$ of the personnel became infected within 6 weeks. This underlies the health risks to health care workers attending to Covid-19 patients. There is the need to research more effective ways of preventing transmission of highly infectious pathogens to health personnel during outbreaks. Provision of a daily log symptom monitoring is a useful tool for ensuring that health care workers monitor their health status, allowing infection to be identified early. Persistent exposures to infected sources, long shift duration, work pressure and work intensity were noted to influence HCWs infection rate.

\section{Conflicts of Interest}

The authors declare no conflicts of interest regarding the publication of this paper.

\section{References}

[1] Adhikari, S.P., Meng, S., Wu, Y.J., Mao, Y.P., Ye, R.X., Wang, Q.Z., Sun, C., et al. 
(2020) Epidemiology, Causes, Clinical Manifestation and Diagnosis, Prevention and Control of Coronavirus Disease (COVID-19) during the Early Outbreak Period: A Scoping Review. Infectious Diseases of Poverty, 9, 1-12. https://doi.org/10.1186/s40249-020-00646-X

[2] Nigeria Centre for Disease Control (2020) https://ncdc.gov.ng/news/227/update-of-corona-virus-disease-in-nigeria

[3] Zhang, M., Zhou, M., Tang, F., Wang, Y., Nie, H., Zhang, L. and You, G. (2020) Knowledge, Attitude, and Practice Regarding COVID-19 among Healthcare Workers in Henan, China. Journal of Hospital Infection, 105, 183-187. https://doi.org/10.1016/j.jhin.2020.04.012

[4] Manzoni, P. and Milillo, C. (2020) Covid-19 Mortality in Italian Doctors. The Journal of Infection, 81, e106-e107. https://doi.org/10.1016/j.jinf.2020.05.034

[5] Zhan, M., Qin, Y., Xue, X. and Zhu, S. (2020) Death from COVID-19 of 23 Health Care Workers in China. New England Journal of Medicine, 382, 2267-2268. https://doi.org/10.1056/NEJMc2005696

[6] Chersich, M.F., Gray, G., Fairlie, L., Eichbaum, Q., Mayhew, S., Allwood, B., English, R., et al (2020) COVID-19 in Africa: Care and Protection for Frontline Healthcare Workers. Globalization and Health, 16, 1-6. https://doi.org/10.1186/s12992-020-00574-3

[7] Amoo, O.S., Ohihoin, A.G., Musa, A.Z., Idighe, I., Ige, F., Giwa-Tubosun, T., Oloko, S., et al. (2020) Implementation of a Modified Drive-Through Sampling Strategy for SARS-CoV-2 the Nigerian Experience. Pan African Medical Journal, 35, 107. https://doi.org/10.11604/pamj.supp.2020.35.2.24319

[8] Infection Prevention and Control Assessment Framework at the Facility Level. https://www.who.int/infection-prevention/tools/core-components/IPCAF-facility.P $\underline{\mathrm{DF}}$

[9] Bseaubein, J. (2020) How South Korea Reined in Coronavirus without Shutting Everything Down. NPR.

https://www.npr.org/sections/goatsandsoda/2020/03/26/821688981/how-south-kore a-reigned-in-the-outbreak-without-shutting-everything-down

[10] (2020) Lagos, Lagos, Nigeria Monthly Weather|AccuWeather. https://www.accuweather.com/en/ng/lagos/4607/april-weather/4607

[11] Coronavirus Disease (COVID-19) Training: Online Training. https://www.who.int/emergencies/diseases/novel-coronavirus-2019/training/onlinetraining

[12] Sabetian, G., Moghadami, M., Hashemizadeh Fard Haghighi, L., Shahriarirad, R., Fallahi, M.J., Asmarian, N. and Moeini, Y.S. (2021) COVID-19 Infection among Healthcare Workers: A Cross-Sectional Study in Southwest Iran. Virology Journal, 18, 58. https://doi.org/10.1186/s12985-021-01532-0

[13] Lai, X., Wang, M., Qin, C., Li, T., Ran, L.S., Chen, D.Q., et al. (2020) Coronavirus Disease 2019 (COVID-2019) Infection among Health Care Workers and Implications for Prevention Measures in a Tertiary Hospital in Wuhan, China. JAMA Network Open, 3, e209666. https://doi.org/10.1001/jamanetworkopen.2020.9666 